\title{
Fluorescent Chemosensors for Fluoride Anion Based on Naphthalene Derivatives Bearing Two Urea Groups
}

\author{
Masaru Sakamaki and Yasumasa Fukushima* \\ Department of Biological Applied Chemistry, Graduate School of Engineering, \\ Toyo University, 2100 Kujirai, Kawagoe, Saitama 350-8585, Japan \\ *E-mail:fukusima_m@toyo.jp
}

Novel fluorescent chemosensors (2,3-NE, 2,3-NE-Ac) containing naphthalene as fluorescent signaling moiety and urea groups as anion binding sites were designed and synthesized, and their spectral behaviors toward various anions were investigated. The chemosensor 2,3-NE was prepared by reacting 2,3-diaminonaphthalene with ethyl isocyanatoacetate and 2,3-NE-Ac was obtained by saponifying 2,3-NE with $\mathrm{Na}_{2} \mathrm{CO}_{3}$. The chemosensor 2,3-NE was shown to signal selectively the detection of fluoride ion in the fluorescence spectra in $\mathrm{CH}_{3} \mathrm{CN} / \mathrm{DMSO}$ (9:1, v/v) even extremely low concentration of fluoride anion. The significant spectral changes indicate that 2,3-NE can be a highly sensitive fluorescent chemosensor for fluoride anion by highly efficient quenching between the excited state of the fluorophore and fluoride ion. Job plot studies revealed that the presence of fluoride ion induces the formation of a 2:1 complex between 2,3-NE and fluoride ion. In addition, the binding mode with fluoride ion was investigated by ${ }^{1} \mathrm{H}$ NMR experiments. The chemosensor 2,3-NE-Ac also recognized fluoride anion, but is approximately 10-fold lower in sensitivity than 2,3-NE by the influence of the carboxylic acid groups. 2,3-NE can become a promising chemosensor for fluoride ion with high sensitivity and selectivity.

Keyword: fluoride anion chemosensor, fluorescence spectroscopy, urea group, naphthalene derivatives

\section{Introduction}

Development of fluorescent chemosensors for biologically important substrates is one of the current research topics in analytical chemistry and biochemistry [1-4]. Recently, considerable attention has been focused on the construction of chemosensors capable of selectively and sensitively detecting anions in a convenient, inexpensive, quantitative, rapid manner because anions play important roles in wide range of chemical, biological, and medical processes [5-8]. Among the range of biologically important anions, fluoride ion, is of particular interest owing to its established role in dental care application and osteoporosis [9], which associated with nerve gases, in the analysis of drinking water, and the refinement of uranium used in nuclear weapon manufacture [10-12]. Furthermore, overexposure to fluoride ion can also result in fluorosis which induces nephrotoxic changes in human body and leads to urolithiasis. Accordingly, it is significant to design and synthesize the selective and sensitive sensor for fluoride anion because the determination of fluoride anionic species and its concentration is of interest in many areas.

Fluorescent chemosensors are attracting extensive attention for their high sensitivity and conveniently fast evaluation. The photophysical properties of fluorescent chemosensors such as fluorescence intensity, wavelength, and fluorescence lifetime change through various mechanisms such as photoinduced electron transfer (PET) [13], intramolecular charge transfer [14], excimer/exciplex formation [15], metal-ligand charge transfer [16], fluorescence resonance energy transfer (FRET) [17], and an increase of their rigidity when they recognize a guest species. In general, fluorescent chemosensors based on PET mechanism displays a ratiometric response which can qualify the guest concentration by using the

\footnotetext{
Received July 22, 2013

Accepted September 5, 2013
} 
change of fluorescence intensity.

Commonly, a typical fluorescence anion chemosensor is the compound containing a fluorophore and a binding unit for anion $[18,19]$. In this study, we selected naphthalene derivatives as a fluorophore because they have been extensively investigated for fluorescence probes due to their inherent photochemical characteristics. The urea groups have been proven to be an excellent and effective hydrogen bonding donor for anion recognition [20-22]. We have reported convenient synthesis and evaluation of two novel fluorescent chemosensors (2,3-NE, 2,3-NE-Ac), shown in Scheme 1, bearing two urea groups at 2,3 positions of naphthalene for the selective recognition of $\mathrm{F}^{-}$. Both 2,3-NE and 2,3-NE-Ac would be expected to have the ability to bind effectively with $\mathrm{F}^{-}$by two urea groups located near to each other. In addition, this paper describes the comparison between the ester groups of 2,3-NE and the carboxyl groups of 2,3-NE-Ac with regard to sensitivity and selectivity of recognition for $\mathrm{F}^{-}$.
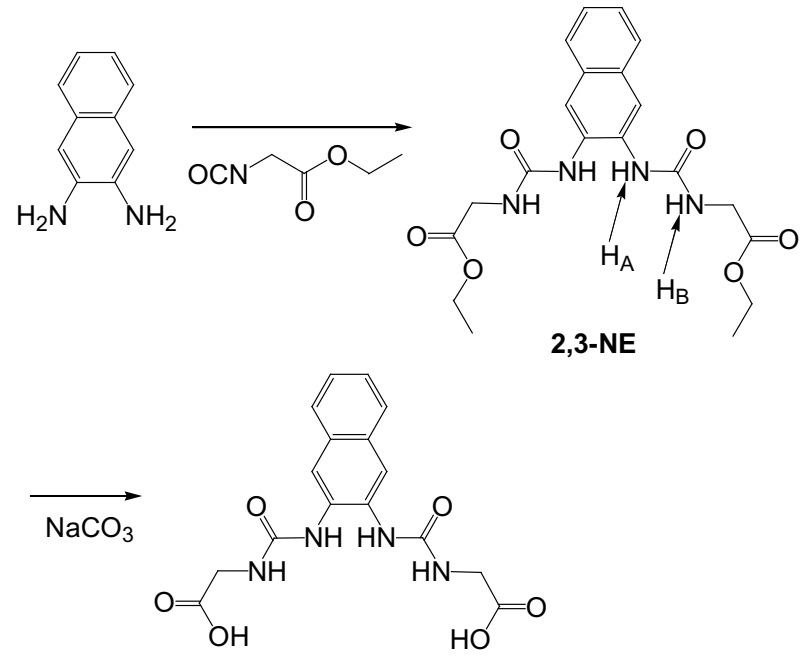

2,3-NE-Ac

Scheme 1. Synthetic routes of chemosensors 2,3-NE and 2,3-NE-Ac

\section{Experimental}

\subsection{Materials}

All reagents and solvents for synthesis were of high purity commercially available and were used without further purification. The tetraethylammonium salts of all the halogen anions $\left(\mathrm{F}^{-}, \mathrm{Cl}^{-}, \mathrm{Br}^{-}\right.$, and $\left.\mathrm{I}^{-}\right)$and the tetrabutylammonium salts of the other anions $\left(\mathrm{HSO}_{4}^{-}, \mathrm{NO}_{3}^{-}\right.$, and $\left.\mathrm{H}_{2} \mathrm{PO}_{4}{ }^{-}\right)$ were purchased from Tokyo Chemical Industry Co.,Ltd. and Sigma-Aldrich Chemical Co., respectively.
2.2. Syntheses of ethyl-2-\{3-[N'-(ethoxycarbonylmethyl)ureido]naphthalen-2-yl-carbamoylamino $\}$ acetate (2,3-NE) and 2-\{3-[N'-(carbonylmethyl)ureido]naphthalen-2-yl-carbamoylamino $\}$ acetic acid (2,3-NE-Ac)

The chemosensors 2,3-NE and 2,3-NE-Ac were synthesized by the synthetic routes shown in Scheme 1. According to literature procedures [23, 24], 2,3-NE was obtained by reacting 2,3-diaminonaphthalene with ethyl isocyanatoacetate in dry $\mathrm{CHCl}_{3}$ at room temperature for $11 \mathrm{~h}$. After completion of reaction, the white powder was filtered and washed with acetone and water for removing impurities, giving analytical pure 2,3-NE in $90 \%$ yield, respectively. ${ }^{1} \mathrm{H}$ NMR $\left(400 \mathrm{MHz}, \mathrm{DMSO}-d_{6}\right)^{\prime} 1.22\left(\mathrm{t}, 6 \mathrm{H}, \mathrm{CH}_{3}\right), 3.94(\mathrm{~d}$, $\left.4 \mathrm{H}, \mathrm{CH}_{2}\right), 4.15\left(\mathrm{q}, 4 \mathrm{H}, \mathrm{CH}_{2}\right), 7.01(\mathrm{t}, 2 \mathrm{H}, \mathrm{NH})$, $7.34(\mathrm{~m}, 2 \mathrm{H}$, aromatic protons $), 7.72(\mathrm{~m}, 2 \mathrm{H}$, aromatic protons), $8.06(\mathrm{~s}, 2 \mathrm{H}$, aromatic protons), 8.25 (s, 2H, NH).

The synthesis of 2,3-NE-Ac was performed by saponifying 2,3-NE with $\mathrm{Na}_{2} \mathrm{CO}_{3}$ at $90^{\circ} \mathrm{C}$ for $1 \mathrm{~h}$, the solution was acidified with $1 \mathrm{~N} \mathrm{HCl}$ to give white precipitate, which was filtered and washed with acetone, giving in $80 \%$ yield. ${ }^{1} \mathrm{H}$ NMR (400 MHz, DMSO- $\left.d_{6}\right)^{\prime} 3.84\left(\mathrm{~d}, 4 \mathrm{H}, \mathrm{CH}_{2}\right), 6.91(\mathrm{t}, 2 \mathrm{H}$, $\mathrm{NH}), 7.33(\mathrm{~m}, 2 \mathrm{H}$, aromatic protons), $7.72(\mathrm{~m}, 2 \mathrm{H}$, aromatic protons), $8.06(\mathrm{~s}, 2 \mathrm{H}$, aromatic protons), $8.24(\mathrm{~s}, 2 \mathrm{H}, \mathrm{NH})$.

\subsection{Apparatus}

${ }^{1} \mathrm{H}$ NMR spectra were recorded on a JEOL $400 \mathrm{MHz}$ spectrometer by using TMS as an internal standard. Fluorescence measurements were carried out on a JASCO F-750 fluorescence spectrometer with a quartz cuvette of $1 \mathrm{~cm}$ path length.

\subsection{Procedures}

Stock solutions $(1000 \mu \mathrm{M}, 100 \mu \mathrm{M}$, and $10 \mu \mathrm{M})$ of all the anions in $\mathrm{CH}_{3} \mathrm{CN}-\mathrm{DMSO}(9: 1 \mathrm{v} / \mathrm{v})$ were prepared. A stock solution of respective chemosensor $(100 \mu \mathrm{M})$ was prepared in $\mathrm{CH}_{3} \mathrm{CN}-\mathrm{DMSO}(9: 1, \mathrm{v} / \mathrm{v})$. All fluorescence measurements were performed with the chemosensor-anion mixture solutions prepared by adding an appropriate aliquot of each anion stock solution to $500 \mu \mathrm{L}$ of each chemosensor solution and diluting the solution to $5 \mathrm{~mL}$ with $\mathrm{CH}_{3} \mathrm{CN}$-DMSO $(9: 1, \quad \mathrm{v} / \mathrm{v})$ under ambient temperature.

${ }^{1} \mathrm{H}$ NMR titration experiments were carried out in the $\mathrm{CD}_{3} \mathrm{CN}-\mathrm{DMSO}-d_{6} \quad(9: 1, \quad \mathrm{v} / \mathrm{v})$ solution prepared by adding increasing amount of fluoride anion to 2,3-NE solution (1 mM). 


\section{Results and Discussion}

\subsection{Anion sensing studies for $\mathbf{2 , 3 - N E}$}

The chemosensor 2,3-NE carries hydrogen bonding donors on urea gruops, while $\mathrm{F}^{-}$possesses a negative charge. 2,3-NE can bind with $\mathrm{F}^{-}$through electrostatic interactions. Figure 1a shows that the investigation of the binding properties of 2,3-NE with $\mathrm{F}^{-}$was performed by measuring the changes of fluorescence spectra of 2,3-NE when titrated with $\mathrm{F}^{-}$in $\mathrm{CH}_{3} \mathrm{CN}-\mathrm{DMSO}(9: 1, \mathrm{v} / \mathrm{v})$. When 2,3-NE is excited at $327 \mathrm{~nm}$, it emits fluorescence ranging from 335 to $450 \mathrm{~nm}$ with a peak maxima and a shoulder around 365 and $350 \mathrm{~nm}$, respectively. The fluorescence intensity of $10 \mu \mathrm{M}$ of $\mathbf{2 , 3 - N E}$ decreased with increasing the concentration of $\mathrm{F}^{-}$ with a blue shift about $5 \mathrm{~nm}$ from 365 to $370 \mathrm{~nm}$. As shown in Figure 1b, the fluorescence intensity decreases gradually up to $2 \mu \mathrm{M}$ of $\mathrm{F}^{-}$concentration and decays rapidly ranging in concentration from 2 to $5 \mu \mathrm{M}$. Above $5 \mu \mathrm{M}$ of $\mathrm{F}^{-}$, the fluorescence intensities are almost flat. The significant fluorescence spectral changes were observed even extremely low concentration of $\mathrm{F}^{-}$. The results indicate that 2,3-NE can be a highly sensitive fluorescent chemosensor for $\mathrm{F}^{-}$. A possible mechanism for the fluorescence quenching of 2,3-NE is attributed to its aggregation derived from 2,3-NE and $\mathrm{F}^{-}$electrostatic complex, resulting in a highly efficient quenching by $\mathrm{F}^{-}$.

In order to study binding selectivity of $\mathbf{2 , 3 - N E}$, fluorescence titrations have been carried out by adding other anions such as $\mathrm{Cl}^{-}, \mathrm{Br}^{-}, \mathrm{I}^{-}, \mathrm{HSO}_{4}^{-}$, $\mathrm{NO}_{3}^{-}, \mathrm{H}_{2} \mathrm{PO}_{4}^{-}$beside $\mathrm{F}^{-}$. As shown in Figure 2, no significant fluorescence intensity change of 2,3-NE occurred in the presence of other anions except in the case of $\mathrm{F}^{-}$. An obvious fluorescence spectral change was observed after adding $\mathrm{F}^{-}$from 0.1 to 1 equivalent as shown in Figure 1a, whereas no detectable spectral changes were observed even with addition of 10 equivalent of other anions. These results suggest that 2,3-NE can recognize $\mathrm{F}^{-}$ with excellent selectivity over the other anions due to the stronger interactions between $\mathrm{F}^{-}$and the urea groups of 2,3-NE. Considering the difference in electronegativity, the ability of $\mathrm{F}^{-}$binding to hydrogens of the urea groups is much stronger than the other anions.

A Job plot was obtained according the method of continuous variations in order to gain an insight into the stoichiometry of the 2,3-NE-F ${ }^{-}$complex [25]. The total concentration of $\mathbf{2 , 3}-\mathbf{N E}$ and $\mathrm{F}^{-}$was constant $(20 \mu \mathrm{M})$, with a continuous variable molar fraction of 2,3-NE $\left([2,3-\mathrm{NE}] /\left[2,3-\mathrm{NE}+\mathrm{F}^{-}\right]\right)$. Figure 3 shows the Job plot of 2,3-NE and $\mathrm{F}^{-}$in $\mathrm{CH}_{3} \mathrm{CN}-\mathrm{DMSO}(9: 1, \mathrm{v} / \mathrm{v})$. Interestingly, this
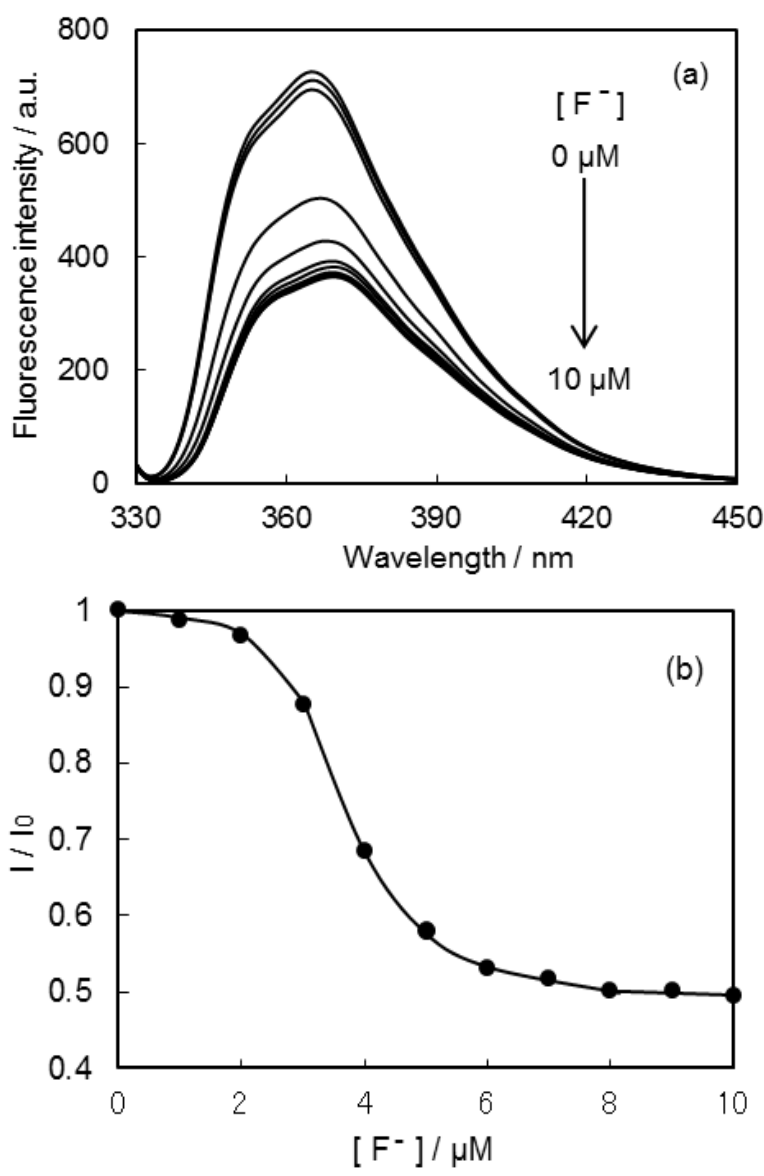

Figure 1. (a) Fluorescent titration spectra of 2,3-NE (10 $\mu \mathrm{M})$ in $\mathrm{CH}_{3} \mathrm{CN}-\mathrm{DMSO}(9: 1, \mathrm{v} / \mathrm{v})$ in the presence of $\mathrm{F}^{-}$at different concentrations, $\left[\mathrm{F}^{-}\right]=0,1,2,3,4,5,6,7$, and $10 \mu \mathrm{M}$ upon excitation at $327 \mathrm{~nm}$. (b) Normalized fluorescence intensity of $\mathbf{2 , 3 - N E}(10 \mu \mathrm{M})$ as a function of $\mathrm{F}^{-}$concentration. $\mathrm{I}_{0}$ and $\mathrm{I}$ denote the fluorescence intensity at $365 \mathrm{~nm}$ in the absence and in the presence of $\mathrm{F}^{-}$, respectively.

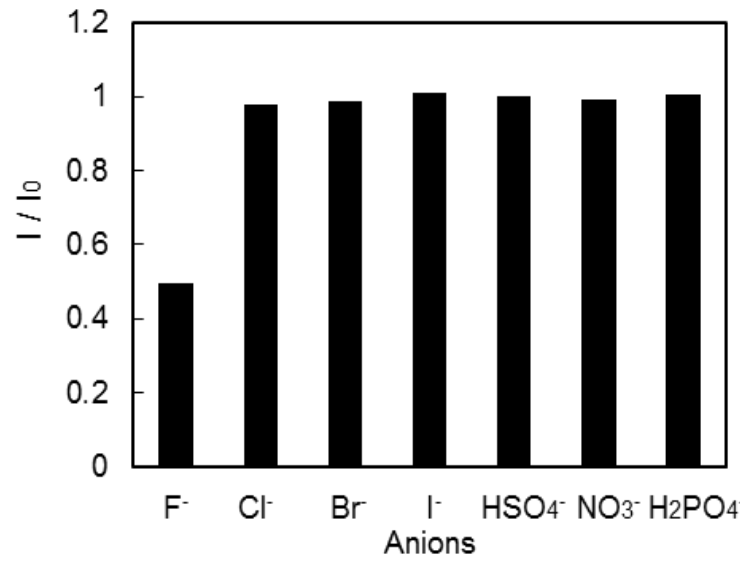

Figure 2. Normalized fluorescence intensity of 2,3-NE $(10 \mu \mathrm{M})$ at $365 \mathrm{~nm}$ upon addition of various anions (10 equiv. $100 \mu \mathrm{M})$ in $\mathrm{CH}_{3} \mathrm{CN}$-DMSO $(9: 1, \mathrm{v} / \mathrm{v})$ excited at $327 \mathrm{~nm} . \mathrm{I}_{0}$ and I denote the fluorescence intensity at 365 $\mathrm{nm}$ in the absence and in the presence of an anion, respectively. 
approach reveals that the relative $\mathbf{2 , 3 - N E}$ and $\mathrm{F}^{-}$ complex concentration clearly approached a maximum when the molar function of 2,3-NE was 0.7, suggesting the formation of a 2:1 stoichiometry complex between $\mathbf{2 , 3 - N E}$ and $\mathrm{F}^{-}$.

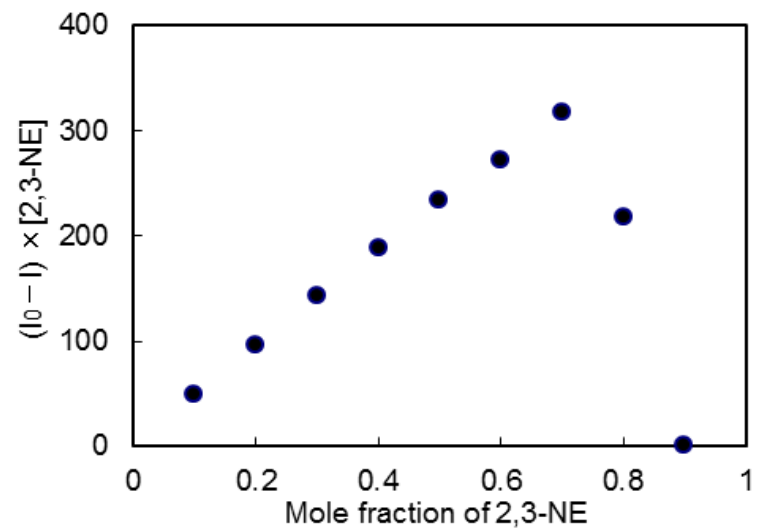

Figure 3. Job plot for 2,3-NE with $\mathrm{F}^{-}$determined by fluorescence measurement in $\mathrm{CH}_{3} \mathrm{CN}-\mathrm{DMSO}(9: 1, \mathrm{v} / \mathrm{v})$, $[\mathbf{2}, \mathbf{3}-\mathrm{NE}]+\left[\mathrm{F}^{-}\right]=20 \mu \mathrm{M}$.

For a complex of 2:1 stoichiometry, the relation in Eq. (1) could be derived to estimate the association constant between $\mathbf{2 , 3 - N E}$ and $\mathrm{F}^{-} . K_{21}$ denotes the observed association constant for $\mathrm{GH}_{2}$ complex formation [26]. $[G]_{\text {tot }}$ and $[H]_{\text {tot }}$ mean the total concentrations of host $(\mathbf{2 , 3 - N E})$ and guest $\left(\mathrm{F}^{-}\right)$, respectively. \pm is fluorescent intensity ratio, $\pm=(I$ $\left.-I_{0}\right) /\left(I_{\mathrm{i}}-I_{0}\right)$, where $I$ is the fluorescence intensity of concentration dependence, and $I_{0}$ and $I_{\mathrm{i}}$ are the fluorescence intensities at zero and infinite anion concentrations.

$$
\begin{aligned}
& G+2 H \stackrel{K_{21}}{=} G H_{2} \\
& {[G]_{\text {tot }}=\frac{a}{2 K_{21}(1-a)^{2}[H]_{\text {tot }}}+\frac{\alpha[H]_{\text {tot }}}{2}} \\
& \alpha=\frac{I-I_{0}}{I_{1}-I_{0}}
\end{aligned}
$$

From fluorescence titration experiments, the estimation of the association constant $\left(K_{21}\right)$ is carried out in Microsoft Office Excel using nonlinear curve fitting based on the solver algorithm, giving $K_{21}=5.2 \times 10^{11} \mathrm{M}^{-2}$. This value suggests 2,3-NE makes complex with $\mathrm{F}^{-}$strongly.

To look into the anion binding properties of chemosensor 2,3-NE with $\mathrm{F}^{-},{ }^{1} \mathrm{H}$ NMR titration experiments in $\mathrm{CD}_{3} \mathrm{CN}-\mathrm{DMSO}-d_{6}(9: 1, \mathrm{v} / \mathrm{v})$ were performed. Upon addition of small amount $(0.1$ to 0.5 equiv) of $\mathrm{F}^{-}$, the ${ }^{1} \mathrm{H}$ NMR spectra of $2,3-\mathrm{NE}$ changed dramatically as shown in Figure 4. The two peaks at $8.5 \mathrm{ppm}\left(\mathrm{H}_{\mathrm{A}}\right)$ and $6.5 \mathrm{ppm}\left(\mathrm{H}_{\mathrm{B}}\right)$, could be attributed to the $\mathrm{NH}$ moieties protons, broadened and exhibited remarkable downfield shifts in $\mathrm{H}_{\mathrm{A}}$ protons from $8.5 \mathrm{ppm}$ to $9.5 \mathrm{ppm}$ and $\mathrm{H}_{\mathrm{B}}$ protons from $6.5 \mathrm{ppm}$ to $7.6 \mathrm{ppm}$. These results reveal that protons in the urea groups play important role in the interaction with fluoride anion due to the formation of the hydrogen bonding in the solution [27]. Moreover, the signals for protons of naphthalene from $7.3 \mathrm{ppm}$ to 8.0 ppm shifted downfield with the changes in their spin-spin splitting by the addition of $\mathrm{F}^{-}$. The naphthalene protons may be influenced by the other 2,3-NE in the 2:1 complex between 2,3-NE and $\mathrm{F}^{-}$.

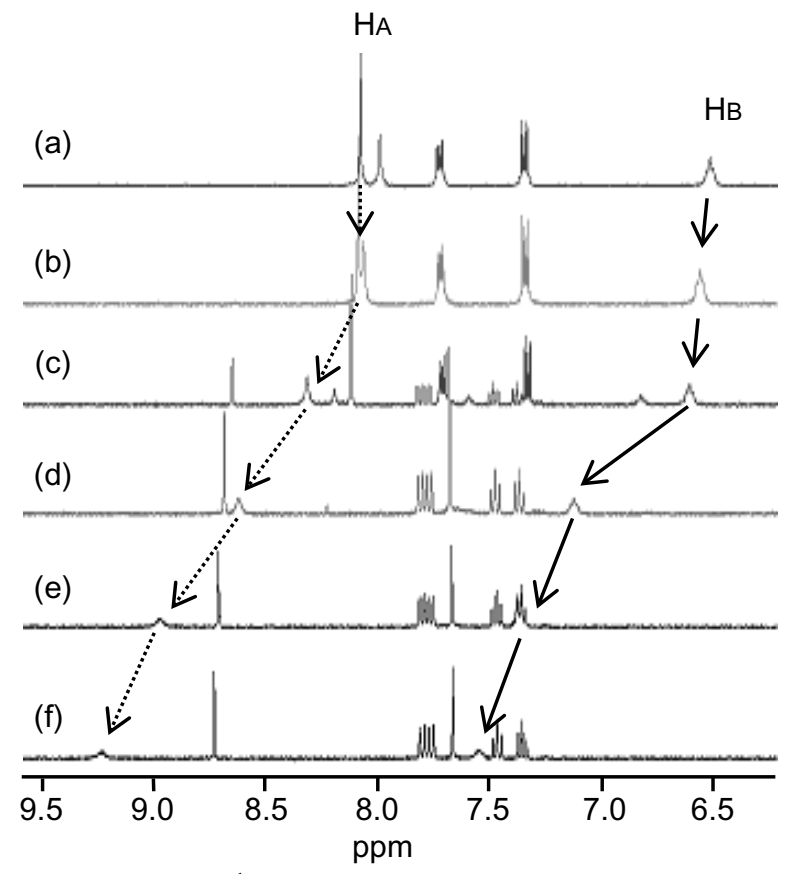

Figure 4. Partial ${ }^{1} \mathrm{H}$ NMR spectra of 2,3-NE (1 mM) in $\mathrm{CD}_{3} \mathrm{CN}-\mathrm{DMSO}-d_{6}(9: 1, \mathrm{v} / \mathrm{v})$ upon the addition of molar equiv. of $\mathrm{F}^{-}$(a) none, (b) 0.1 equiv., (c) 0.2 equiv., (d) 0.3 equiv., (e) 0.4 equiv., (f) 0.5 equiv.

The selectivity of 2,3-NE for $\mathrm{F}^{-}$could be rationalized on the basis of the electronegativity as well as complementary shape between the host and the anion guest. The presence of multiple interactions between host and guest would be essential for the construction of high sensitive and selective sensors. $\mathrm{F}^{-}$can bind to the four urea groups of 2,3-NE due to formation of the 2:1 complex between $\mathbf{2 , 3 - N E}$ and $\mathrm{F}^{-}$. Consequently, the binding mode of 2,3-NE with $\mathrm{F}^{-}$was proposed as depicted in Figure 5.

\subsection{Anion sensing studies for 2,3-NE-Ac}

The fluorescence spectra were also recorded from a solution $(10 \mu \mathrm{M})$ of 2,3-NE-Ac in $\mathrm{CH}_{3} \mathrm{CN}-\mathrm{DMSO}(9: 1, \mathrm{v} / \mathrm{v})$ on excitation at $327 \mathrm{~nm}$. 


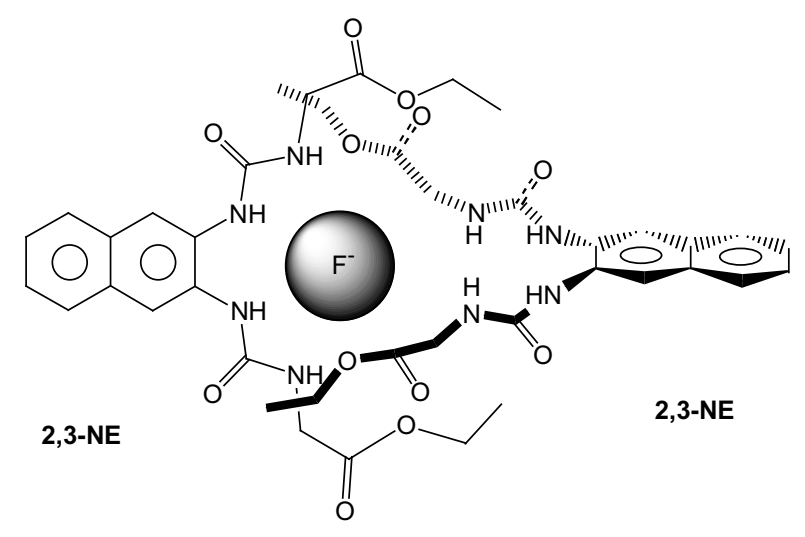

Figure 5. The possible binding mode for a 2:1 stoichiometry complex between of 2,3-NE with $\mathrm{F}^{-}$.

Figure 6a shows that the fluorescence spectrum for 2,3-NE-Ac is almost comparable with that for 2,3-NE in the absence of $\mathrm{F}^{-}$. As shown in Figure 6b, the fluorescence intensity of 2,3-NE-Ac at $365 \mathrm{~nm}$ was gradually decreased with the increase in concentration up to $60 \mu \mathrm{M}$ of $\mathrm{F}^{-}$, whereas the fluorescence intensities remain almost unchanged above $60 \mu \mathrm{M}$ of $\mathrm{F}^{-}$. 2,3-NE-Ac is approximately 10-fold lower than 2,3-NE in the sensitivity for $\mathrm{F}^{-}$ because of the carboxylic acid groups nearby urea groups of 2,3-NE-Ac.

The Job plot of 2,3-NE-Ac with $\mathrm{F}^{-}$was measured to determine the stoichiometry. Figure 7 shows the maximum is at a molar fraction $\left([2,3-N E-A c] /\left[2,3-N E-A c+F^{-}\right]\right)$of 0.5 , which means the formation of 2,3-NE-Ac and $\mathrm{F}^{-}$complex with a 1:1 ratio. These observations indicate that 2,3-NE-Ac binds to $\mathrm{F}^{-}$with the 1:1complex because it could not approach each other owing to the carboxylic acid groups unlike 2,3-NE.

For a complex of 1:1 stoichiometry, Benesi-Hildebrand method based on Eq. (2) was used for estimating association constant $\left(K_{1}\right)$ [28, 29].

$\Delta F$ means the difference of fluorescence yields between the complex and 2,3-NE-Ac. Symbol $[G]_{0}$ and $[H]_{0}$ mean concentration of host (2,3-NE-Ac) and guest $\left(\mathrm{F}^{-}\right)$, respectively. $I$ and $I_{0}$ are the fluorescence intensity at $365 \mathrm{~nm}$ of concentration dependence and fluorescence intensity at zero guest concentration, respectively. The association constant of 2,3-NE-Ac for $\mathrm{F}^{-}$was determined to be $1.1 \times 10^{4} \mathrm{M}^{-1}$ with a good linear relationship by plotting $1 /\left(I-I_{0}\right)$ and $1 /[H]_{0}$.

$$
G+H \stackrel{K_{1}}{=} G H
$$

$$
\frac{1}{I-I_{0}}=\frac{1}{\Delta F[H]_{0}}+\frac{1}{\Delta F[H]_{0}[G]_{0} K_{1}}
$$

$\Delta F$ means the difference of fluorescence yields between the complex and 2,3-NE-Ac. Symbol $[G]_{0}$ and $[H]_{0}$ mean concentration of host (2,3-NE-Ac) and guest $\left(\mathrm{F}^{-}\right)$, respectively. $I$ and $I_{0}$ are the fluorescence intensity at $365 \mathrm{~nm}$ of concentration dependence and fluorescence intensity at zero guest concentration, respectively. The association constant of 2,3-NE-Ac for $\mathrm{F}^{-}$was determined to be $1.1 \times 10^{4} \mathrm{M}^{-1}$ with a good linear relationship by plotting $1 /\left(I-I_{0}\right)$ and $1 /[H]_{0}$.
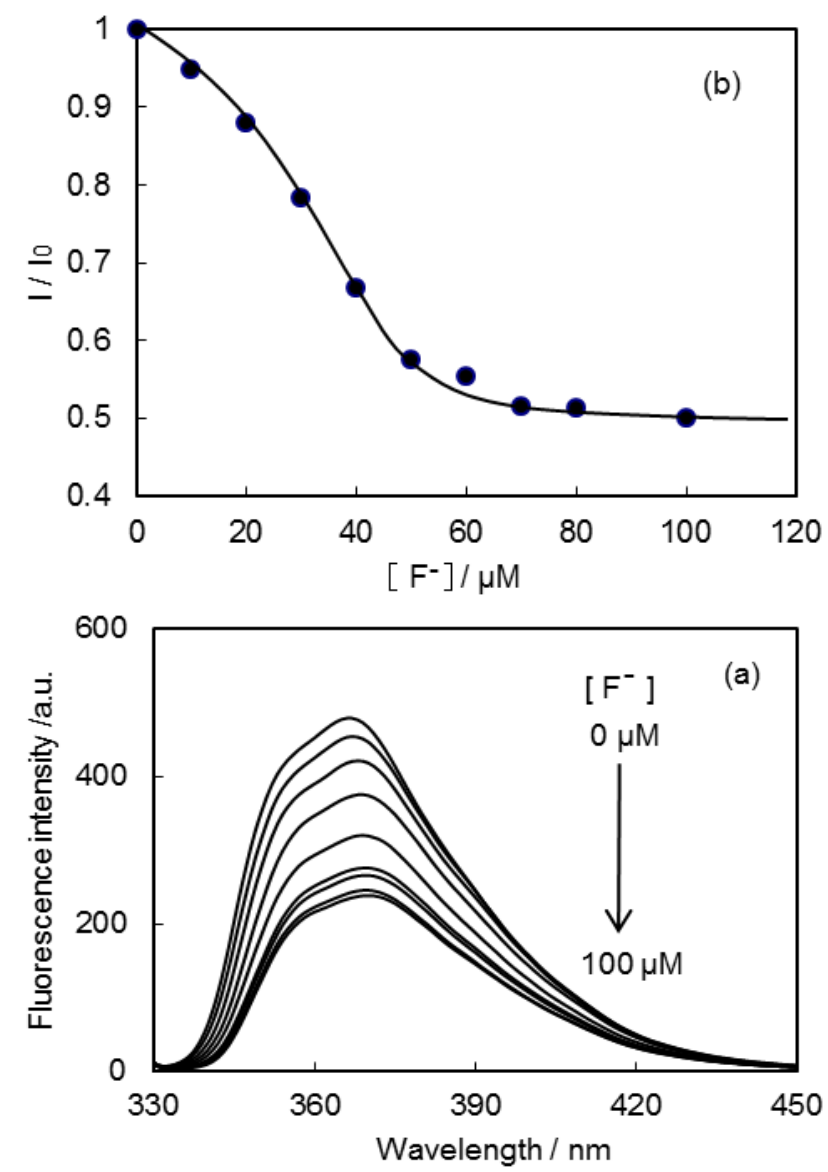

Figure 6. (a) Fluorescent titration spectra of 2,3-NE-Ac $(10 \mu \mathrm{M})$ in $\mathrm{CH}_{3} \mathrm{CN}-\mathrm{DMSO}(9: 1, \mathrm{v} / \mathrm{v})$ in the presence of $\mathrm{F}^{-}$at different concentrations, $\left[\mathrm{F}^{-}\right]=0,10,20,30,40,50$, 60,70 , and $100 \mu \mathrm{M}$ upon excitation at $327 \mathrm{~nm}$. (b) Normalized fluorescence intensity of $2,3-N E(10 \mu \mathrm{M})$ as a function of $\mathrm{F}^{-}$concentration. $\mathrm{I}_{0}$ and $\mathrm{I}$ denote the fluorescence intensity at $365 \mathrm{~nm}$ in the absence and in the presence of $\mathrm{F}^{-}$, respectively.

\section{Conclusion}

In summary, we have developed a highly sensitive and selective fluorescent fluoride anion chemosensor 2,3-NE based on naphthalene with two urea groups as the recognition moiety. For 2,3-NE in $\mathrm{CH}_{3} \mathrm{CN}-\mathrm{DMSO}(9: 1, \mathrm{v} / \mathrm{v})$, the fluorescence was quenched effectively by fluoride 


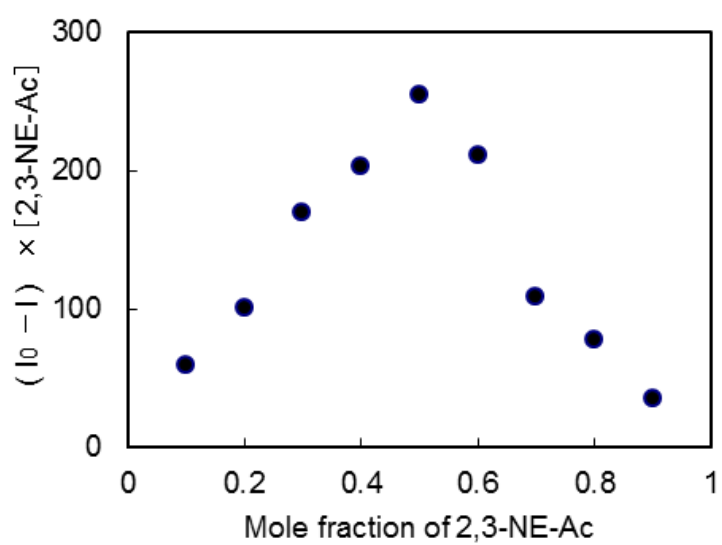

Figure 7. Job plot for 2,3-NE-Ac with $\mathrm{F}^{-}$determined by fluorescence measurement in $\mathrm{CH}_{3} \mathrm{CN}-\mathrm{DMSO}(9: 1, \mathrm{v} / \mathrm{v})$, $[2,3-\mathrm{NE}-\mathrm{Ac}]+\left[\mathrm{F}^{-}\right]=20 \mu \mathrm{M}$.

anion in a range of 0 to $5 \mu \mathrm{M}$. Job plot studies revealed the formation of a $2: 1$ stoichiometry complex between 2,3-NE and fluoride anion. NMR titration studies showed that the urea groups play important roles in recognizing fluoride anion by the electrostatic interactions. The two urea groups located near to each other for 2,3-NE would cause significant advantage in terms of the detection limit and selectivity.

\section{References}

1. R.Joseph, and C.P.Rao, Chem. Rev., 111, (2011), 4658.

2. L.Pu, Chem. Rev., 104, (2004), 1687.

3. E.V.Anslyn, J. Org. Chem., 72, (2007), 687.

4. X.Xu and M.P.Doyle, Inorg. Chem., 50, (2011), 7610.

5. P.D.Beer and P.A.Gale, Angew. Chem. Int. Ed., 40, (2001), 486.

6. R.Martinez-Manez and F.Sanceron, Chem. Rev., 103, (2003), 4419.

7. V.Bhalla, R.Kumar, M.Kumar, and A.Dhir, Tetrahedron, 63, (2007), 11153.

8. H.Kim and J.Kang, Tetrahedron Lett., 46, (2005), 5443.

9. M.Kleerekoper, Endcrinol. Metab. Clin. North. Am., 27, (1998), 441.

10. G.LWaldbott, Clin. Toxicol., 18, (1981), 531.
11. S.Matsuo, K.Kiyomiya, and M.Kurebe, Arch. Toxicol., 72, (1998), 798.

12. M.Laisalmi, H.Kokki, A.Soilleli, H.Markkanen, A.Yli-Hankala, P.Rosenberg, and L.Lindgren, Acta. Anaesthesiol. Scand., 50, (2006), 982.

13. D.H.Vance and A.W.Czarnik, J. Am. Chem. Soc., 116, (1994), 9397.

14. F.Y.Wu and Y.B.Jiang, Chem. Phys. Lett., 355, (2002), 438.

15. S.Nishizawa, Y.Kato, and N.Teramae, J. Am. Chem. Soc., 121, (1999), 9463.

16. P.D.Beer, Acc. Chem. Res., 31, (1998), 71.

17. M.H.Lee, D.T.Quang, H.S.Jung, J.Yoon, C.H.Lee, and J.S.Kim, J. Org. Chem., 72, (2007), 4242.

18. J.Du, M.Hu, J.Fan, and X.Peng, Chem. Soc. Rev., 41, (2012), 4511.

19. T.Gunnlaugsson, M.Glynn, G.M.Tocci, P.E.Kruger, and F.M.Pfeffer, Coord. Chem. Rev., 250, (2006), 3094.

20. D.A.Jose, D.K.Kumar, B.Ganguly, and A.Das, Org. Lett., 6, (2004), 3445.

21. R.M.Duke and T.Gunnlaugsson, Tetrahedron Lett., 48, (2007), 8043.

22. D.Jemerez, R.M.Manez, F.Sancenon, and J.Sato, Tetrahedron Lett., 43, (2002), 2823.

23. M.Kumar, R.Kumar, and V.Bhalla, Tetrahedron, 65, (2009), 4340.

24. J.Shao, Dyes Pigm., 87, (2010), 272.

25. Y.Kubo, M.Kato, Y.Misawa, and S.Tokita, Tetrahedron Lett., 45, (2004), 3769.

26. M.V.Alfimov, A.V.Churakov, Y.V.Fedorova, S.P.Gromov, R.E.Hester, J.A.K.Howard, L.G.Kuz'mina, I.K.Lednev, and J.N.Moore, $J$. Chem. Soc., Perkin Trans. 2., (1997), 2249.

27. F.Han, Y.H.Bao, Z.G.Yang, T.M.Fyles, J.Z.Zhao, X.J.Peng, J.L.Fan, Y.K.Wu, and S.G.Sun, Chem. Eur. J., 13, (2007), 2880.

28. M.Berra, C.Bohne, and J.C.Scaiano, J. Am. Chem. Soc., 112, (1990), 8075.

29. H.Benesi and J.H.Hildebrand, J. Am. Chem. Soc., 71, (1949), 2703. 